\title{
Voltage Control in Smart Grids: An Approach Based on Sensitivity Theory
}

\author{
Morris Brenna $^{1}$, Ettore De Berardinis ${ }^{2}$, Federica Foiadelli ${ }^{1}$, Gianluca Sapienza ${ }^{3}$, Dario Zaninelli ${ }^{1}$ \\ ${ }^{1}$ Politecnico di Milano - Department of Energy, Milan, Italy; ${ }^{2}$ CESI S.p.A., Milan, Italy; ${ }^{3}$ Politecnico di Milano - Department of \\ Energy in Collaboration with ENEL Distribuzione S.p.A., Milan, Italy. \\ Email: gianluca.sapienza@mail.polimi.it
}

Received March $30^{\text {th }}, 2010$; revised May 25 ${ }^{\text {th }}, 2010$; accepted May $31^{\text {st }}, 2010$

\begin{abstract}
Due to the development of Distributed Generation $(D G)$, which is installed in Medium-Voltage Distribution Networks (MVDNs) such as generators based on renewable energy (e.g., wind energy or solar energy), voltage control is currently a very important issue. The voltage is now regulated at the MV busbars acting on the On-Load Tap Changer of the HV/MV transformer. This method does not guarantee the correct voltage value in the network nodes when the distributed generators deliver their power. In this paper an approach based on Sensitivity Theory is shown, in order to control the node voltages regulating the reactive power exchanged between the network and the dispersed generators. The automatic distributed voltage regulation is a particular topic of the Smart Grids.
\end{abstract}

Keywords: Voltage Regulation, Reactive Power Injection, Distributed Generation, Smart Grids, Sensitivity Theory, Renewable Energy

\section{Introduction}

Due to the development of Distributed Generation (DG), which is installed in Medium-Voltage Distribution Networks (MVDNs) such as generators based on renewable energy (e.g., wind energy or solar energy), voltage control is currently a very important issue.

The voltage of MVDNs is now regulated acting only on the On-Load Tap Changer (OLTC) of the HV/MV transformer [1]. The OLTC control is typically based on the compound technique, and this method does not guarantee the correct voltage value in the network nodes when the generators deliver their power [2,3].

When a generator injects power in the network, the voltage tends to rise. In HV networks this phenomenon happens mainly when reactive power is injected, because the resistance is negligible if compared with the inductive reactance [4]. Instead in MVDNs the resistance is not negligible and the result is that an injection of active power also increases the voltage.

In other words the so-called $P \theta-Q V$ decoupling [5], which is a typical of $\mathrm{HV}$ networks, is inexistent in MVDNs. The P variations are "coupled" with the voltage variations.

If no precautions are taken, in particular network conditions the overcome of the maximum admissible voltage can happen in any nodes.
When a generator injects power, the voltage rises in all network nodes, but some nodes are mainly influenced than others by the power injection. This influence can be obtained using a Sensitivity method.

In this paper an approach based on Sensitivity Theory is shown, in order to control the network voltage using the reactive power exchanged between network and the distributed generators. This approach allows to control the voltage in the long term period. Besides, fastdynamic voltage disturbances are not taken into account [6].

After the theoretical analysis, a numerical example is shown, in order to validate the proposed theory.

The proposed method differs from the others used in HV networks analysis, based on the Jacobian Matrix [1,2-4] and its application is easy.

The topological proprieties that results from the theoretical analysis imply that the proposed sensitivity method can be easily implemented in automatic voltage control devices, in order to obtain the distributed voltage regulation.

The automatic voltage regulation in a distributed manner is a typical topic of the Smart Grids context.

The paper is structured in the following way. In Section 1, the proposed voltage control method is shown, and an overview on the voltage profiles with DG, are given. In Section 3, the proposed Sensitivity approach is 
studied, referring to a MV test network, composed by four nodes. Finally, in Section 4, a numerical application is presented, in order to validate the proposed theory.

\section{The Proposed Criteria to Control the Network Voltage with Distributed Generation}

Many methods can be used to control the voltage in network nodes (network voltages). The proposed method varies the reactive power exchanged between the generators and the network while maintaining the OL-TC in a fixed position for a particular load condition.

Let us suppose that the Automatic Voltage Regulator (AVR) that controls the OLTC maintains the MV bus-bar voltage at the rated value (1 p.u.), assuming that the transformer taps are adequate.

For passive grids, when no generators are connected to the MVDN, the voltage profile (VP; i.e., the voltage values along a line) decreases monotonically (see profile a in Figure 1) due to the load absorptions. When the generators are connected and inject power into the MVDN, the nodal voltages increase and the VP is no longer monotonic, as shown in profile b in Figure 1 (profile b). This phenomenon also occurs if generators work at unitary power factor (i.e., only active power is injected due to the non-negligible network resistance) [7].

It is important to note that, in steady-state, the condition maintained at the MV busbar by the AVR decouples the MV feeders, and the result is that each feeder works without the influence of the other lines. In other words, the loads and generators connected to other feeders do not influence the VP of the considered line.

Typically, the generators installed in Smart Grids are based on renewable energy; therefore, their power-time profiles are unknown. Due to the high generated power and a possibly low load condition, the voltage in some nodes can thus exceed the maximum admissible value ( $V_{\max }$; i.e., the voltage threshold [8]) defined by the standards.

Of course the voltage threshold is strictly related with the settings of the voltage relays installed in the network, e.g. at the generator nodes [9].

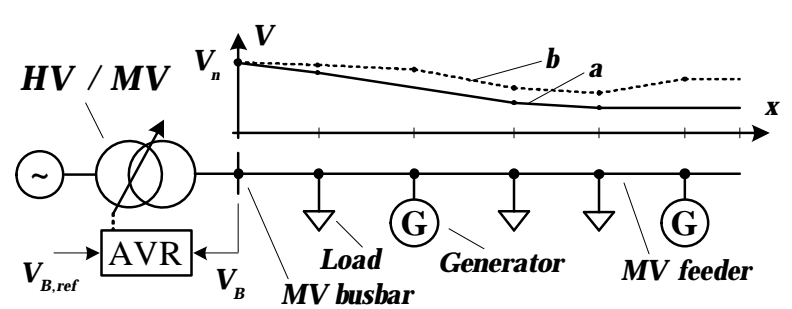

Figure 1. Voltage profiles in a MV feeder with and without Distributed Generators
If the generators are able to control the injected or absorbed reactive power, the network voltage profiles can be modified by acting on the reactive powers. It is clear that each controllable generator needs a Generator Remote Terminal Unit (GRTU) that is connected to a central control system to set the generator reactive power, (i.e., to control the exciter of the synchronous generators [1] or act on the inverter control if the generator is inverter-based) $[10,11]$. In this work, the central control is called the Generator Control Centre (GCC). In addition, we use a hierarchical control structure $[12,13]$.

Let us suppose that the voltage is measured only in the generator nodes by the GRTUs. This assumption does not affect the generality of the proposed method because a Measuring Remote Terminal Unit connected to the GCC can be installed in each node that must be controlled.

When the voltage in the $i^{\text {th }}$ node exceeds $V_{\max }$, the GRTU installed in the same node sends the signal "Voltage Threshold Overall" (VTO) to the GCC using a communication channel. The GCC then selects the generator in the $j^{\text {th }}$ node that has the maximum influence on the voltage of the $i^{\text {th }}$ node, the "Best Generator" (BG), and switches it to the reactive power absorption (RPA) mode. Therefore, the voltage in the $i^{\text {th }}$ node tends to decrease.

The problem is thus to determine the best generator and ensure that the GCC chooses it. In this work, a sensitivity-based method is proposed to select the BG.

Moreover, we suppose that the generators can only be switched in the RPA mode by the GCC by a constant power factor. Therefore, if $P_{j}$ is the active power injectted by the generator connected to the $j^{\text {th }}$ node, then it absorbs the reactive power $Q_{j}=P_{j} \tan \varphi_{j}$ (where $\cos \varphi_{j}$ is the minimum power factor of the generator) when it is switched during RPA. In other words, we assume that no continuous reactive power modulation is possible.

An example of the procedure described above is shown in Figure 2. Let us suppose that load Ld suddenly decreases its power (for example, due to a trip) and $V_{2}$ exceed $V_{\max }$.

The GRTUs of G2 send the signal VTO to the GCC that must choose the BG using the sensitivity method. Assuming that the BG is G1, it will be switched by the

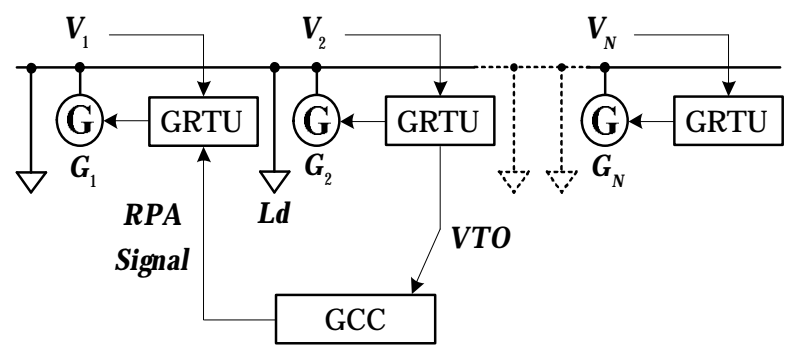

Figure 2. Voltage control using GRTU and GCC 
GCC in the RPA mode; therefore, the reactive power absorbed by $\mathrm{G} 1$ becomes $Q_{1}=P_{1} \tan \varphi_{1}$.

As explained in the following, the GCC must know the reactive power that each controllable generator can absorb in order to choose the BG. We suppose that this information is acquired by the GCC using a polling technique on each GRTU.

\section{The Proposed Sensitivity Approach}

\subsection{Classical Sensitivity Theory Overview}

The classical sensitivity theory used in HV network analysis to perform primary and secondary voltage regulation [14] is based on the Jacobian Matrix and reveals the relationships between the nodal voltages (magnitude and phase) and the nodal power injections (active and reactive). The relationships mentioned above are represented by the following matrix expression [2]:

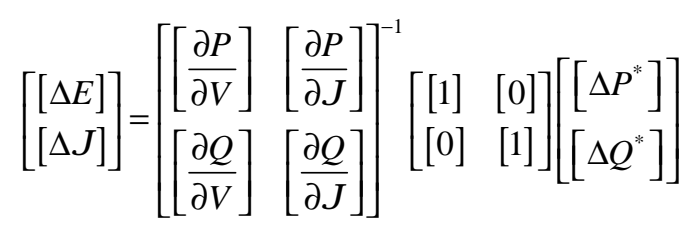

where $[\Delta E]$ and $[\Delta \vartheta]$ are, respectively, the nodal voltage magnitudes (rms) and phase variations corresponding to the nodal active or reactive power injections $\left[\Delta P^{*}\right]$ and $\left[\Delta Q^{*}\right]([1]$ is the identity matrix $)$.

Equation (1) can be rewritten in the following compact form:

$$
\left[\begin{array}{l}
{[\Delta E]} \\
{[\Delta \vartheta]}
\end{array}\right]=[s]\left[\begin{array}{l}
{\left[\Delta P^{*}\right]} \\
{\left[\Delta Q^{*}\right]}
\end{array}\right]
$$

where:

$$
[s] @\left[\begin{array}{ll}
{\left[\frac{\partial P}{\partial V}\right]} & \left.\left[\frac{\partial P}{\partial \vartheta}\right]\right]^{-1}\left[\begin{array}{ll}
{[1]} & {[0]} \\
{[0]} & {[1]}
\end{array}\right]
\end{array}\right.
$$

is the (injection) sensitivity matrix. The method descries above is generally valid, but its computational complexity is too high for practical voltage analysis in MVDNs. For radial networks, only the voltage magnitude is needed to control the nodal voltages. The proposed theory is easier than classical theory, and it is suitable for radial MVDNs.

\subsection{The Proposed Theory}

In this section, the proposed theory for choosing the BG is outlined. The method is first described in general and considers the possibility of reactive power regulation for all nodes.
After the general treatment, the analysis focuses on a realistic network in which the reactive power can only be controlled in some nodes (generator nodes).

Let us consider the network depicted in Figure 3, which is a four-node test MVDN.

The general loads $L d_{1} \ldots L d_{4}$ are represented using constant PQ models. Positive $P$ (or $Q$ ) corresponds to the absorbed power by the load. Negative $P$ (or $Q$ ) corresponds to the injected power in the network (i.e., the general load is really a generator). The per-phase equivalent circuit is shown in Figure 4.

The lines $L_{01} \ldots L_{24}$ are modeled using the RL-direct sequence equivalent circuit [15], but the shunt admittances are neglected. The node 0 represents the MV busbar, which is regulated at a constant voltage value $E_{0}$ by the AVR of the OLTC. This reference voltage coincides with the rated value $E_{0}=V_{n} / \sqrt{3}$.

Because the busbar is regulated at $E_{0}$, we can characterize the generic node $i$ using the difference $V_{0 i}$ between the magnitude of the busbar voltage and the node voltage $E_{i}$. In other words, we can write:

$$
V_{0 i}=E_{0}-E_{i}
$$

In radial networks, (4) can be calculated as the sum of the voltage differences between adjacent nodes from the ith node toward the MV busbar. For example, if $i=3$ (see Figure 4), (4) becomes:

$$
V_{03}=E_{0}-E_{3}
$$

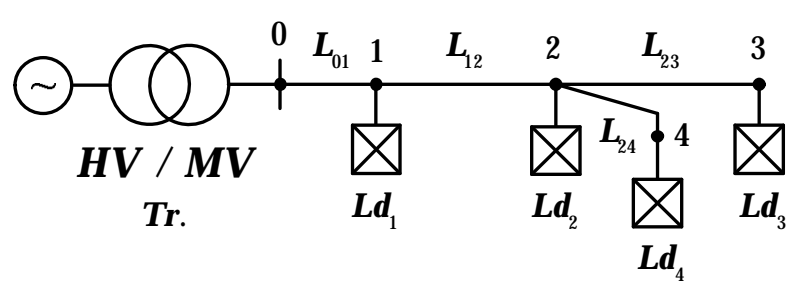

Figure 3. The considered four nodes test MVDN

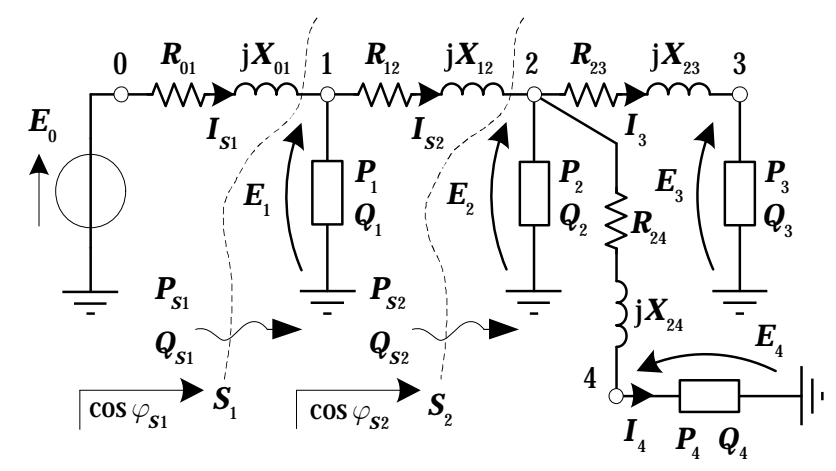

Figure 4. The per-phase equivalent circuit 
By adding and subtracting $E_{1}$ and $E_{2}$ in (5), we obtain:

$$
\begin{aligned}
V_{03} & =\left(E_{0}-E_{1}\right)+\left(E_{1}-E_{2}\right)+\left(E_{2}-E_{3}\right) \\
& =V_{01}+V_{12}+V_{23}
\end{aligned}
$$

where $V_{03}$ is the sum of the voltage differences $V_{01}$, $V_{12}$ and $V_{23}$.

$V_{23}$ can be calculated considering the network parameters and the line power flows as follows:

$$
\begin{aligned}
V_{23} & =E_{2}-E_{3} \\
& =R_{23} I_{3} \cos \varphi_{3}+X_{23} I_{3} \sin \varphi_{3} \\
& =\frac{R_{23} E_{3} I_{3} \cos \varphi_{3}+X_{23} E_{3} I_{3} \sin \varphi_{3}}{E_{3}} \\
& =\frac{R_{23} P_{3}+X_{23} Q_{3}}{E_{3}}
\end{aligned}
$$

where $\cos \varphi_{3}, P_{3}$ and $Q_{3}$ are the power factor and the active and reactive (per-phase) powers of the load $L d_{3}$, respectively. $I_{3}, R_{23}$ and $X_{23}$ are the current, resistance and reactance of the line $L_{3}$.

Normally, the nodal voltages are close to the rated voltage $E_{n}$. Applying this assumption to (7) leads to:

$$
V_{23} \cong \frac{R_{23} P_{3}+X_{23} Q_{3}}{E_{n}}
$$

Similarly, considering nodes 1 and 2, we can write:

$$
\begin{aligned}
V_{12} & =E_{1}-E_{2} \\
& =R_{12} I_{2} \cos \varphi_{S 2}+X_{12} I_{2} \sin \varphi_{S 2} \\
& =\frac{R_{12} E_{2} I_{2} \cos \varphi_{S 2}+X_{12} E_{2} I_{2} \sin \varphi_{S 2}}{E_{2}} \\
& \cong \frac{R_{12} P_{S 2}+X_{12} Q_{S 2}}{E_{n}}
\end{aligned}
$$

where $P_{S 2}$ and $Q_{S 2}$ are the active and reactive powers through the section $S_{2}$ and $\cos \varphi_{S 2}$ is the power factor for the same section. For $P_{S 2}$ and $Q_{S 2}$, we can write:

$$
\begin{aligned}
& P_{S 2}=P_{2}+P_{3}+P_{4}+P_{R 23}+P_{R 24} \\
& Q_{S 2}=Q_{2}+Q_{3}+Q_{4}+Q_{X 23}+Q_{X 24}
\end{aligned}
$$

where $P_{R 23}$ and $P_{R 24}$ are the power losses in $R_{23}$ and $R_{24}$, while $Q_{X 23}$ and $Q_{X 24}$ are the reactive powers absorbed by $X_{23}$ and $X_{24}$. These active and reactive losses are negligible compared to the load powers. Applying this assumption to (9), (10) and (11) leads to:

$$
\begin{aligned}
& P_{S 2} \cong P_{2}+P_{3}+P_{4} \\
& Q_{S 2} \cong Q_{2}+Q_{3}+Q_{4}
\end{aligned}
$$

and:

$$
V_{12} \cong \frac{R_{12}\left(P_{2}+P_{3}+P_{4}\right)+X_{12}\left(Q_{2}+Q_{3}+Q_{4}\right)}{E_{n}}
$$

Finally, the voltage difference $V_{01}$ is:

$$
V_{01}=E_{0}-E_{1} \cong \frac{R_{01} P_{S 1}+X_{01} Q_{S 1}}{E_{n}}
$$

where:

$$
\begin{aligned}
& P_{S 1} \cong P_{1}+P_{2}+P_{3}+P_{4} \\
& Q_{S 1} \cong Q_{1}+Q_{2}+Q_{3}+Q_{4}
\end{aligned}
$$

are the powers through section $S_{1}$.

Using (6) with (15), (9) and (8), we can say that $V_{03}$ is a function of all loads and active and reactive powers, i.e., $P_{1} \ldots P_{4}$ and $Q_{1} \ldots Q_{4}$. The same observation is valid for $E_{3}$ :

$$
E_{3}=E_{0}-V_{03}=E_{0}-\left(V_{01}+V_{12}+V_{23}\right)
$$

because $E_{0}$ is constant. In other words, we can write:

$$
E_{3}=f\left(P_{1}, \ldots, P_{4}, Q_{1}, \ldots, Q_{4}\right)
$$

Equation (19) shows that an active/reactive power variation (in the general $\mathrm{j}$ node) that is defined as:

$$
\begin{gathered}
\Delta P_{j}=P_{j}^{f}-P_{j}^{0} \\
\Delta Q_{j}=Q_{j}{ }^{f}-Q_{j}{ }^{0}
\end{gathered}
$$

where $P_{j}^{f} \quad\left(Q_{j}^{f}\right)$ and $P_{j}^{0} \quad\left(Q_{j}^{0}\right)$ are the final and initial power values, respectively, produces a voltage variation in node 3 that is defined as:

$$
\Delta E_{3}=E_{3}^{f}-E_{3}^{0}
$$

In this treatment, we only consider the reactive power variations (i.e., $\left.\Delta P_{j}=0\right)$ because we assume that only the reactive power can be used to control the node voltages.

The variation $\Delta E_{3}$ can be calculated by linearizing (19) and considering only the reactive power variations. In particular, we can write:

$$
\begin{gathered}
\Delta E_{3}=\frac{\partial E_{3}}{\partial Q_{1}} \Delta Q_{1}+\frac{\partial E_{3}}{\partial Q_{2}} \Delta Q_{2}+ \\
+\frac{\partial E_{3}}{\partial Q_{3}} \Delta Q_{3}+\frac{\partial E_{3}}{\partial Q_{4}} \Delta Q_{4}
\end{gathered}
$$

The terms $\partial E_{i} / \partial Q_{j}$ in (23) indicate the "gain" from the voltage variation $\Delta E_{i}$ in node $i$ when a reactive power variation $\Delta Q_{j}$ occurs in node $j$. In other words, they are sensitivity terms.

According to (18), we can obtain: 


$$
\begin{aligned}
& \frac{\partial E_{3}}{\partial Q_{2}}=-\frac{X_{01}+X_{12}}{E_{n}} \\
& \frac{\partial E_{3}}{\partial Q_{3}}=-\frac{X_{01}+X_{12}+X_{23}}{E_{n}} \\
& \frac{\partial E_{3}}{\partial Q_{4}}=-\frac{X_{01}+X_{12}}{E_{n}}
\end{aligned}
$$

Substituting equation group (24) into (23) has important implications. If we have a reactive injection in any node, i.e., $\Delta Q_{j}<0$ (in this case $j=1 \ldots 4$ ), then $\Delta E_{3}>0$ in node 3 (i.e., the voltage increases). Then, if we were to reduce the voltage in any node, we must abs- orb reactive power from the network (i.e., $\Delta Q_{j}>0$ ) by using, for example, the distributed generators.

If the above analysis that focuses on node 3 is extended to all network nodes, (23) has a general matrix relationship:

$$
\left[\begin{array}{l}
\Delta E_{1} \\
\Delta E_{2} \\
\Delta E_{3} \\
\Delta E_{4}
\end{array}\right]=\left[\begin{array}{llll}
\frac{\partial E_{1}}{\partial Q_{1}} & \frac{\partial E_{1}}{\partial Q_{2}} & \frac{\partial E_{1}}{\partial Q_{3}} & \frac{\partial E_{1}}{\partial Q_{4}} \\
\frac{\partial E_{2}}{\partial Q_{1}} & \frac{\partial E_{2}}{\partial Q_{2}} & \frac{\partial E_{2}}{\partial Q_{3}} & \frac{\partial E_{2}}{\partial Q_{4}} \\
\frac{\partial E_{3}}{\partial Q_{1}} & \frac{\partial E_{3}}{\partial Q_{2}} & \frac{\partial E_{3}}{\partial Q_{3}} & \frac{\partial E_{3}}{\partial Q_{4}} \\
\frac{\partial E_{4}}{\partial Q_{1}} & \frac{\partial E_{4}}{\partial Q_{2}} & \frac{\partial E_{4}}{\partial Q_{3}} & \frac{\partial E_{4}}{\partial Q_{4}}
\end{array}\right]\left[\begin{array}{l}
\Delta Q_{1} \\
\Delta Q_{2} \\
\Delta Q_{3} \\
\Delta Q_{4}
\end{array}\right]
$$

which in a compact form yields:

$$
[\Delta E]=\left[s_{Q}\right][\Delta Q]
$$

where $\left[s_{Q}\right]$ is the reactive sensitivity matrix, $[\Delta Q]$ is the reactive power-variations vector and $[\Delta E]$ is the nodal voltages vector.

Calculating the partial derivatives contained in $\left[s_{Q}\right]$, we have Equation (27).

After analyzing this form of (27), we can say that this matrix can be built using the following inspection rule:

"The element $i, j$ is the arithmetic sum of the reactance of the branches in which both the powers absorbed by node $i$ and node $j$ flow multiplied by $-1 / E_{n}$ ".

For example, in (27), the element 2, 4 is

$$
-\left(X_{01}+X_{12}\right) / E_{n}
$$

because the powers delivered by node 2 and node 4 flow in branches 01 and 12 .

\subsection{The Choice of the Best Generator}

The BG is the generator that has the greatest influence on node $i$, which is the node where the voltage exceeds the threshold.

Thus, after analyzing (25), we can say that the BG is the generator that maximizes the following product, which we call the "sensitivity product":

$$
\frac{\partial E_{i}}{\partial Q_{j}} \Delta Q_{j}
$$

For example, if the node with a voltage that exceeds $V_{\text {max }}$ is $i=2$ and the BG is connected to node $j=4$, the sensitivity product $\left(\partial E_{2} / \partial Q_{4}\right) \Delta Q_{4}$ is the highest compared to the other products contained in row 2 of the sensitivity matrix. In addition, in order to choose the BG, it is necessary to evaluate the single products (28) of the row that represents node $i$. Thus, the value $\Delta Q_{j}$ is needed and is acquired as the GCC polls the GRTUs, as stated previously.

The procedure described above suggests a way of defining the "sensitivity table" $\left[T_{S}\right]$ that contains the single sensitivity products. For the MVDN represented in Figure 4, $\left[T_{S}\right]$ takes the following form

$$
\left[\begin{array}{llll}
\frac{\partial E_{1}}{\partial Q_{1}} \Delta Q_{1} & \frac{\partial E_{1}}{\partial Q_{2}} \Delta Q_{2} & \frac{\partial E_{1}}{\partial Q_{3}} \Delta Q_{3} & \frac{\partial E_{1}}{\partial Q_{4}} \Delta Q_{4} \\
\frac{\partial E_{2}}{\partial Q_{1}} \Delta Q_{1} & \frac{\partial E_{2}}{\partial Q_{2}} \Delta Q_{2} & \frac{\partial E_{2}}{\partial Q_{3}} \Delta Q_{3} & \frac{\partial E_{2}}{\partial Q_{4}} \Delta Q_{4} \\
\frac{\partial E_{3}}{\partial Q_{1}} \Delta Q_{1} & \frac{\partial E_{3}}{\partial Q_{2}} \Delta Q_{2} & \frac{\partial E_{3}}{\partial Q_{3}} \Delta Q_{3} & \frac{\partial E_{3}}{\partial Q_{4}} \Delta Q_{4} \\
\frac{\partial E_{4}}{\partial Q_{1}} \Delta Q_{1} & \frac{\partial E_{4}}{\partial Q_{2}} \Delta Q_{2} & \frac{\partial E_{4}}{\partial Q_{3}} \Delta Q_{3} & \frac{\partial E_{4}}{\partial Q_{4}} \Delta Q_{4}
\end{array}\right]
$$

Row $i$ represents the node in which we want to control the voltage, and column $j$ represents the nodes in which we can control the reactive power. The BG is the generator connected to node $j$ that has the maximum absolute value of the sensitivity product in position $i, j$. By finding the maximum sensitivity product in row $i$,

$$
\left[s_{Q}\right]=-\frac{1}{E_{n}}\left[\begin{array}{cccc}
X_{01} & X_{01} & X_{01} & X_{01} \\
X_{01} & X_{01}+X_{12} & X_{01}+X_{12} & X_{01}+X_{12} \\
X_{01} & X_{01}+X_{12} & X_{01}+X_{12}+X_{23} & X_{01}+X_{12} \\
X_{01} & X_{01}+X_{12} & X_{01}+X_{12} & X_{01}+X_{12}+X_{24}
\end{array}\right]
$$


we automatically choose the BG because the location corresponds to column $j$ of the maximum sensitivity product.

It is clear that, for a general network with $\mathrm{N}$ nodes, the sensitivity table takes the following form:

$$
\left[\begin{array}{cccc}
\frac{\partial E_{1}}{\partial Q_{1}} \Delta Q_{1} & \frac{\partial E_{1}}{\partial Q_{2}} \Delta Q_{2} & \cdots & \frac{\partial E_{1}}{\partial Q_{N}} \Delta Q_{N} \\
\frac{\partial E_{2}}{\partial Q_{1}} \Delta Q_{1} & \cdots & \cdots & \cdots \\
\cdots & \cdots & \cdots & \cdots \\
\frac{\partial E_{N}}{\partial Q_{1}} \Delta Q_{1} & \cdots & \cdots & \frac{\partial E_{N}}{\partial Q_{N}} \Delta Q_{N}
\end{array}\right]
$$

It is important to note that, if it is not possible to regulate the reactive power (e.g., if in that node there is a load or a non-controllable generator) in a node $j$, then $\Delta Q_{j}=0$ and, consequently, the sensitivity product in the position $i, j$ of the sensitivity table is 0 .

Comparing (29) with (25), we can say that each element $i, j$ of $\left[T_{S}\right]$ represents the line-to-ground voltage variation in node $i$ when a reactive power variation occurs in node $j$.

In the following section, a numerical example of the sensitivity method application is shown.

\section{Application of the Proposed Method}

The network considered in this numerical application is represented in Figure 5.

During normal network operation, we have four generators and eight loads. The generator and load characteristics are summarized in Table $\mathbf{1}$ ( $S$ is the apparent power) and Table 2, respectively (three-phase powers are represented in these tables).

We suppose that the generators normally operate with a unitary power factor (i.e., no reactive power is injected in the nodes).

The per-kilometer reactance of the cable lines is $x=$ $0.17 \Omega / \mathrm{km}$, which is a typical value for Italian MVDNs. The line lengths and parameters are summarized in Table 3.

Let us suppose that each generator is connected to its GRTU that measures the nodal voltage and communicates with the GCC. Moreover, let us suppose that $G_{5}$ cannot regulate the reactive power because it is not designed for this purpose. The MV busbar is regulated at the rated voltage ( 1 p.u.), which is $20 \mathrm{kV}$ (line-to-line). In this example, the voltage threshold $V_{\max }$ is 1.05 p.u.

Using load-flow software, we calculated the voltage $E$ in the generator nodes (nodes 4, 5, 6, and 7) for normal network operation. The results are shown in Figure 6 (Normal Operation).

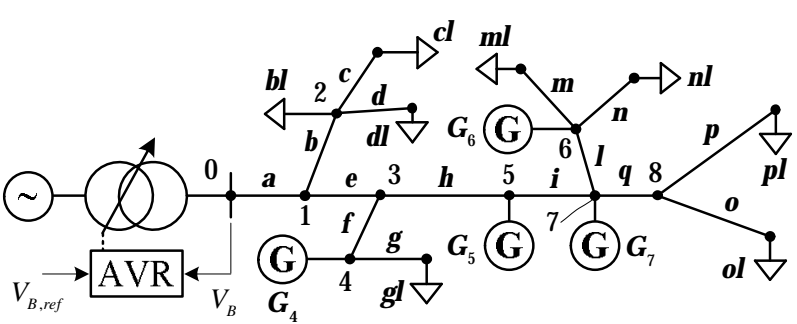

Figure 5. The network considered in the numerical application

Table 1. Loads characteristics

\begin{tabular}{ccccc}
\hline Load & $S[M V A]$ & $c o s$ & $P[M W]$ & $Q[M V A R]$ \\
\hline$b l, n l$ & 2 & 0.95 & 1.9 & 0.62 \\
$c l$ & 7 & 0.92 & 6.44 & 2.74 \\
$d l$ & 3 & 0.92 & 2.76 & 1.18 \\
$g l$ & 2.08 & 0.95 & 1.99 & 0.62 \\
$m l$ & 2.58 & 0.93 & 2.4 & 0.94 \\
$o l$ & 1.98 & 0.96 & 1.9 & 0.57 \\
$p l$ & 1.5 & 0.92 & 1.38 & 0.59 \\
\hline
\end{tabular}

Table 2. Generators characteristics

\begin{tabular}{cc}
\hline Generator & $P[M W]$ \\
\hline$G_{4}$ & 6 \\
$G_{5}$ & 1.75 \\
$G_{6}$ & 4.5 \\
$G_{7}$ & 3.75 \\
\hline
\end{tabular}

Table 3. Lines parameters

\begin{tabular}{ccc}
\hline Line Name & $L[k m]$ & $X[\Omega]$ \\
\hline $\mathrm{a}, \mathrm{l}, \mathrm{q}$ & 2 & 0.34 \\
$\mathrm{~b}, \mathrm{f}, \mathrm{g}, \mathrm{i}, \mathrm{m}, \mathrm{n}$ & 1 & 0.17 \\
$\mathrm{c}, \mathrm{d}, \mathrm{o}, \mathrm{p}$ & 0.5 & 0.085 \\
$\mathrm{e}$ & 15 & 2.55 \\
$\mathrm{~h}$ & 5 & 0.85 \\
\hline
\end{tabular}

If line $b$ trips (e.g., due to a fault), loads $b l, c l$ and $d l$ are cut off from the supply, which causes the voltage to increase in the network. In particular, if the load-flow is re-computed to take into account the new network configuration, we obtain the results shown in Figure 6 (Tripped Line).

It is important to note that, if the voltage exceeds the maximum threshold $V_{\max }$ in node 5, the GRTU connected to $G_{5}$ sends the VTO signal to the GCC that must choose the BG using the sensitivity table.

We suppose that the three-phase reactive powers absorbable by each generator that were collected from the 
last poll are those summarized in Table 4, which also contains the corresponding power factors $\cos \varphi$. To calculate the sensitivity table, we need the single-phase powers. Therefore, the reactive powers shown in Table 4 have to be divided by three. It is important to note that the reactive powers calculated this way correspond to $\Delta Q_{j}$ because $Q_{j}{ }^{0}$ is zero (see (21)). The $\Delta Q_{j}$ values are shown in Table 5.

The voltage exceeds the threshold in node 5. Thus, we only consider the fifth row of the sensitivity table. According to the inspection rule mentioned above, this row is as follows:

$$
\left[\begin{array}{llllllll}
0 & 0 & 0 & t_{S 5,4} & t_{S 5,5} & t_{S 5,6} & t_{S 5,7} & 0
\end{array}\right]
$$

where the single sensitivity products $t_{S i, j}$ are:

$$
\begin{gathered}
t_{S 5,4}=-\frac{1}{E_{n}}\left(X_{a}+X_{e}\right) \Delta Q_{4}=-164.53 \mathrm{~V} \\
t_{S 5,5}=-\frac{1}{E_{n}}\left(X_{a}+X_{e}+X_{h}\right) \Delta Q_{5}=0 \mathrm{~V}
\end{gathered}
$$

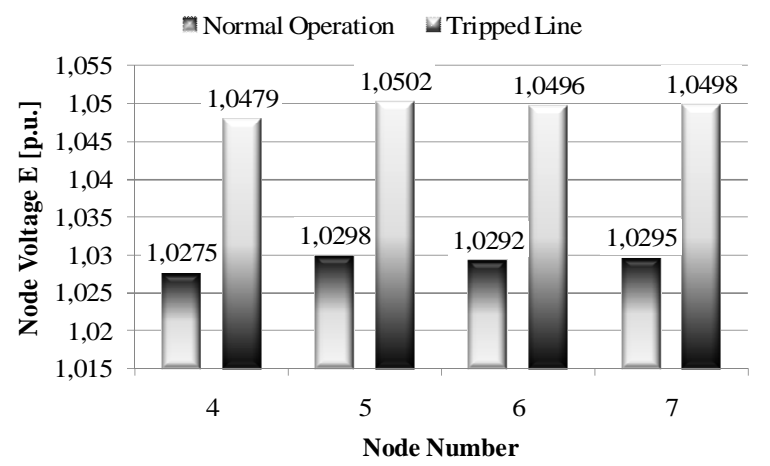

Figure 6. Load-Flow results with the Network Normal Operation

Table 4. Reactive powers absorbable by the generators

\begin{tabular}{ccc}
\hline Generator & $Q[$ MVAR $]$ & $\cos \varphi$ \\
\hline$G_{4}$ & 1.97 & 0.95 \\
$G_{5}$ & 0.00 & 1 \\
$G_{6}$ & 0.91 & 0.98 \\
$G_{7}$ & 1.23 & 0.95 \\
\hline
\end{tabular}

Table 5. Reactive Power Variations in the Generator Nodes

\begin{tabular}{cc}
\hline Generator & $\Delta Q_{j}[M V A R]$ \\
\hline$G_{4}$ & 0.66 \\
$G_{5}$ & 0.00 \\
$G_{6}$ & 0.30 \\
$G_{7}$ & 0.41 \\
\hline
\end{tabular}

$$
\begin{aligned}
t_{S 5,6} & =-\frac{1}{E_{n}}\left(X_{a}+X_{e}+X_{h}\right) \Delta Q_{6} \\
& =-98.65 \mathrm{~V} \\
t_{S 5,7} & =-\frac{1}{E_{n}}\left(X_{a}+X_{e}+X_{h}\right) \Delta Q_{7} \\
& =-133.07 \mathrm{~V}
\end{aligned}
$$

The maximum sensitivity product (in absolute value) corresponds to generator 4 , (i.e., $j=4$ ). Thus, the BG is $G_{4}$.

Equation (32) provides important information. If $G_{4}$ performs the considered reactive power variation, the line-to-ground voltage variation in node 5 is:

$$
\Delta E_{5}=-164.53 \mathrm{~V} \rightarrow-0.0142 \text { p.u. }
$$

Then, considering (22) (rewritten for node 5), and $E_{5}{ }^{0}=1.0502$ p.u. from Figure 6, we can say that the voltage value after the reactive power variation is:

$$
E_{5}{ }^{f}=E_{5}{ }^{0}+\Delta E_{5}=1.036 \text { p.u. }
$$

which is less than the voltage threshold $V_{\max }$.

Equation (37) shows the theoretical result obtained using the proposed method. We checked this value using load-flow software:

$$
E_{5}{ }_{\text {load flow }}=1.034 \text { p.u. }
$$

The percentage error between (38) and (37) is:

$$
\varepsilon_{\%}=\frac{E_{5}{ }^{f} \text { load flow }-E_{5}{ }^{f}}{E_{5}{ }^{f} \text { load flow }} \cdot 100=-0.19 \%
$$

which is negligible and demonstrates the validity of the proposed approach.

\section{Conclusions}

The proposed sensitivity method allows the voltage within network acting on single generators to be regulated by choosing the most effective generator on the controlled node (i.e., the Best Generator). This is a very important feature in grids that have distributed generation (e.g., in a Smart Grid context).

The proposed method uses a topological approach. Moreover, the sensitivity table can be constructed automatically.

In addition to the BG choice, the proposed method also evaluates the voltage in all network nodes after a reactive power variation.

After choosing the BG, but before its commutation during RPA, it is possible to verify that the voltage variation in the other nodes is tolerable for the connected loads. Moreover, it is necessary to verify that the threshold settings of the voltage relay installed in the same nodes. 
When a generator is switched during RPA, it works with a non-unitary power factor; the reactive power flow increases along the lines and increases the power loss [16].

This phenomenon is negligible in HV networks because the line resistance is typically smaller than the line reactance, but is important to consider in MV networks.

Therefore, if network analysis reveals that the RPAswitching produces high losses, voltage control using the reactive power variation must only be used for temporary voltage variation mitigation (i.e., during emergency conditions).

The possible future develops of this work could be focused on the optimization of the forecasted power-time profiles of the loads and generators applying both the se nsitivity approach and distributed voltage measurement.

\section{REFERENCES}

[1] R. Marconato, "Electric Power Systems," Vol. 2, CEI, Milano, 2008.

[2] P. Kundur, "Power System Stability and Control," McGrawHill, New York, 1994.

[3] Y. Rosales Hernandez and T. Hiyama, "Distance Measure Based Rules for Voltage Regulation with Loss Reduction", Journal of Electromagnetic Analysis and Applications (JEMAA), Vol. 1, No. 2, June 2009, pp. 85-91.

[4] F. Saccomanno, "Electric Power Systems" Wiley-Interscience IEEE Press, Piscataway, 2003.

[5] G. Andersson, "Modeling and Analysis of Electric Power Systems" Lecture 227-0526-00, ITET ETH Zürich, Zürich, 2008.

[6] Y. Abdel-Rady, I. Mohamed and E. F. El-Saadany "A Control Scheme for PWM Voltage-Source DistributedGeneration Inverters for Fast Load-Voltage Regulation and Effective Mitigation of Unbalanced Voltage Disturbances" IEEE Transactions on Industrial Electronics, Vol. 55, No. 5, May 2008, pp. 2072-2084.

[7] P. M. S. Carvalho, P. F. Correia and L. A. F. M. Ferreira, "Distributed Reactive Power Generation Control for Vol- tage Rise Mitigation in Distribution Networks," IEEE Transactions on Power Systems, Vol. 23, No. 2, 2008, pp. 766-772.

[8] P. N. Vovos, A. E. Kiprakis, A. R. Wallace and G. P. Harrison, "Centralized and Distributed Voltage Control: Impact on Distributed Generation Penetration," IEEE Transactions on Power Systems, Vol. 22, No. 1, 2007, pp. 476-483.

[9] P. M. Anderson, "Power System Protection," IEEE Press, Piscataway, 1999.

[10] N. Mohan, T. M. Undeland and W. P. Robbins, "Power Electronics: Converters, Applications, and Design", Wiley, 1995.

[11] M. H. Rashid, "Power Electronics Handbook", Academic Press-Elsevier, 2007.

[12] L. L. Grigsby, "Electric Power Generation, Transmission and Distribution," CRC Press-Taylor \& Francis Group, Boca Raton, 2006.

[13] F. A. Viawan and D. Karlsson, "Coordinated Voltage and Reactive Power Control in the Presence of Distributed Generation," PES General Meeting - Conversion and Delivery of Electrical Energy in the 21st Century, IEEE, Pittsburgh, 2008, pp. 1-6.

[14] S. Corsi, "Wide Area Voltage Regulation \& Protection" 2009 IEEE Bucharest Power Tech Conference, Bucharest, June 28 -July 2, Bucharest, pp. 1-7.

[15] A. Gandelli, S. Leva and A. P. Morando, "Topological Considerations on the Symmetrical Components Transformation", IEEE Transactions on Circuits and Systems I: Fundamental Theory and Applications, Vol. 47, No. 8, August 2000, pp. 1202-1211.

[16] H. M. Ayres, L. C. P. da Silva, W. Freitas, M. C. de Almeida and V. F. da Costa, "Evaluation of the Impact of Distributed Generation on Power Losses by Using a Sensitivity-Based Method," IEEE Power \& Energy Society General Meeting, Calgary, 2009, pp. 1-6.

[17] A. Kishore and E. F. Hill, "Static optimization of Reactive Power Sources by use of Sensitivity Parameters", IEEE Transactions on Power Apparatus and Systems, Vol. PAS-90, No. 3, 1971, pp. 1166-1173. 